\title{
Two powerful theorems in Clifford analysis
}

\author{
Fred Brackx \\ Clifford Research Group, Department of Mathematical Analysis, Faculty of Engineering, Ghent University, \\ Galglaan 2, B-9000 Gent, Belgium
}

\begin{abstract}
Two useful theorems in Euclidean and Hermitean Clifford analysis are discussed: the Fischer decomposition and the Cauchy-Kovalevskaya extension.

Keywords: Clifford analysis

PACS: (MSC 2000) 30G35
\end{abstract}

\section{INTRODUCTION}

Recently, when constructing orthogonal bases of spaces of polynomials in Euclidean and Hermitean Clifford analysis using ideas stemming from group representation theory, more particularly the well-known construction of the Gel'fand-Tsetlin basis for irreducible modules of classical Lie groups (see [22, 8]), it became apparent that two basic theorems are really indispensable in this approach: the Fischer decomposition and the Cauchy-Kovalevskaya extension. Of both theorems the classical version and the Clifford analysis versions are enlightened. They not only are powerful tools in Clifford analysis, they are also nice illustrations of how Clifford analyis, in a direct and elegant way, generalizes to higher dimension the concept of holomorphy in the complex plane and at the same times refines harmonic analysis.

\section{BASICS OF CLIFFORD ANALYSIS}

Let $\left(e_{1}, \ldots, e_{m}\right)$ be an orthonormal basis of $\mathbb{R}^{m}$, then multiplication in the real Clifford algebra $\mathbb{R}_{m}$ and in the complex Clifford algebra $\mathbb{C}_{m}=\mathbb{R}_{m} \otimes \mathbb{C}$ is governed by the rule $e_{\alpha} e_{\beta}+e_{\beta} e_{\alpha}=-2 \delta_{\alpha \beta}, \alpha, \beta=1, \ldots, m$, whence both Clifford algebras are generated additively by the elements $e_{A}=e_{j_{1}} \ldots e_{j_{h}}$, where $A=\left\{j_{1}, \ldots, j_{h}\right\} \subset\{1, \ldots, m\}$, with $1 \leq j_{1}<j_{2}<\cdots<j_{h} \leq m$, and $e_{\emptyset}=1$.

In Euclidean Clifford analysis (see e.g. $[3,15,19,20]$ ) the central notion is that of a monogenic function, i.e. a null solution of the so-called Dirac operator $\partial_{\underline{X}}=\sum_{\alpha=1}^{m} e_{\alpha} \partial_{X_{\alpha}}$.

In the books [23, 13] and the series of papers [24, 9, 1, 2, 4, 16, 17] so-called Hermitean Clifford analysis has emerged as a refinement of Euclidean Clifford analysis. The framework for Hermitean Clifford analysis is introduced by means of a complex structure, i.e. an $\mathrm{SO}(m)$-element $J$ with $J^{2}=-\mathbf{1}$ (see $[1,2]$ ). So, the dimension is forced to be even: $m=2 n$. Usually $J$ is chosen to act upon the generators of $\mathbb{C}_{2 n}$ as $J\left[e_{j}\right]=-e_{n+j}$ and $J\left[e_{n+j}\right]=e_{j}, j=1, \ldots, n$. By means of the projection operators $\pm \frac{1}{2}(\mathbf{1} \pm i J)$ associated to $J$, first the Witt basis elements $\left(\mathfrak{f}_{j}, \mathfrak{f}_{j}^{\dagger}\right)_{j=1}^{n}$ for $\mathbb{C}_{2 n}$ are obtained: $\mathfrak{f}_{j}=\frac{1}{2}(\mathbf{1}+i J)\left[e_{j}\right]=\frac{1}{2}\left(e_{j}-i e_{n+j}\right)$ and $\mathfrak{f}_{j}^{\dagger}=-\frac{1}{2}(\mathbf{1}-i J)\left[e_{j}\right]=-\frac{1}{2}\left(e_{j}+i e_{n+j}\right), j=1, \ldots, n$, satisfying the relations $\mathfrak{f}_{j} \mathfrak{f}_{k}+\mathfrak{f}_{k} \mathfrak{f}_{j}=\mathfrak{f}_{j}^{\dagger} \mathfrak{f}_{k}^{\dagger}+\mathfrak{f}_{k}^{\dagger} \mathfrak{f}_{j}^{\dagger}=0$ and $\mathfrak{f}_{j} \mathfrak{f}_{k}^{\dagger}+\mathfrak{f}_{k}^{\dagger} \mathfrak{f}_{j}=\delta_{j k}, j, k=1, \ldots, n$. Next, a vector $\left(x_{1}, \ldots, x_{n}, y_{1}, \ldots, y_{n}\right) \in \mathbb{R}^{2 n}$ is identified with $\underline{X}=\sum_{j=1}^{n}\left(e_{j} x_{j}+e_{n+j} y_{j}\right)$, producing the Hermitean variables $\underline{z}=\frac{1}{2}(\mathbf{1}+i J)[\underline{X}]=\sum_{j=1}^{n} \mathfrak{f}_{j} z_{j}$ and $\underline{z}^{\dagger}=-\frac{1}{2}(\mathbf{1}-i J)[\underline{X}]=\sum_{j=1}^{n} \mathfrak{f}_{j}^{\dagger} \bar{z}_{j}$, expressed in the complex variables $z_{j}=x_{j}+i y_{j}$ and their conjugates $\bar{z}_{j}=x_{j}-i y_{j}$, $j=1, \ldots, n$. Finally, the Dirac operator $\partial_{\underline{X}}=\sum_{j=1}^{n}\left(e_{j} \partial_{x_{j}}+e_{n+j} \partial_{y_{j}}\right)$ gives rise to the Hermitean Dirac operators $\partial_{\underline{z}}^{\dagger}=\frac{1}{4}(\mathbf{1}+i J)\left[\partial_{\underline{X}}\right]=\sum_{j=1}^{n} \mathfrak{f}_{j} \partial_{\bar{z}_{j}}$ and $\partial_{\underline{z}}=-\frac{1}{4}(\mathbf{1}-i J)\left[\partial_{\underline{X}}\right]=\sum_{j=1}^{n} \mathfrak{f}_{j}^{\dagger} \partial_{z_{j}}$, involving the Cauchy-Riemann operators $\partial_{\bar{z}_{j}}=\frac{1}{2}\left(\partial_{x_{j}}+i \partial_{y_{j}}\right)$ and their conjugates $\partial_{z_{j}}=\frac{1}{2}\left(\partial_{x_{j}}-i \partial_{y_{j}}\right), j=1, \ldots, n$. The Hermitean variables and Dirac operators are isotropic, whence the Laplacian decomposes as $\Delta_{2 n}=4\left(\partial_{z} \partial_{z}^{\dagger}+\partial_{z}^{\dagger} \partial_{z}\right)$, while also $\underline{z} \underline{z}^{\dagger}+\underline{z}^{\dagger} \underline{z}=|\underline{z}|^{2}$.

We take functions with values in an irreducible representation $\mathbb{S}_{n}^{-}$of $\mathbb{C}_{2 n}$, called spinor space, which is realized within $\mathbb{C}_{2 n}$ using a primitive idempotent $I=I_{1} \ldots I_{n}$, with $I_{j}=\mathfrak{f}_{j} f_{j}^{\dagger}, j=1, \ldots, n$. With that choice $\mathbb{S}_{n} \equiv \mathbb{C}_{2 n} I \cong \bigwedge_{n}^{\dagger} I$, 
where $\bigwedge_{n}^{\dagger}$ denotes the Grassmann algebra generated by the $\mathfrak{f}_{j}^{\dagger}$ s, since $\mathfrak{f}_{j} I=0$. Hence $\mathbb{S}_{n}$ decomposes into homogeneous parts as $\mathbb{S}_{n}=\bigoplus_{r=0}^{n} \mathbb{S}_{n}^{(r)}=\bigoplus_{r=0}^{n}\left(\bigwedge_{n}^{\dagger}\right)^{(r)} I$, with $\left(\bigwedge_{n}^{\dagger}\right)^{(r)}=\operatorname{span}_{\mathbb{C}}\left(\mathfrak{f}_{k_{1}}^{\dagger} \wedge \mathfrak{f}_{k_{2}}^{\dagger} \wedge \cdots \wedge \mathfrak{f}_{k_{r}}^{\dagger}:\left\{k_{1}, \ldots, k_{r}\right\} \subset\{1, \ldots, n\}\right)$.

A continuously differentiable function $g$ in an open region $\Omega$ of $\mathbb{R}^{2 n}$, taking values in $\mathbb{S}_{n}$, then is called (left) Hermitean monogenic in $\Omega$ iff it satisfies in $\Omega$ the system $\partial_{\underline{z}} g=0=\partial_{\underline{z}}^{\dagger} g$. A major difference with Euclidean Clifford analysis concerns the underlying group invariance. The fundamental group leaving the Dirac operator $\partial_{\underline{X}}$ invariant is the orthogonal group $\mathrm{O}(m)$, which is doubly covered by the $\mathrm{Pin}(m)$ group of the Clifford algebra. However the system invariance of the Hermitean Dirac operators $\left(\partial_{\underline{z}}, \partial_{\underline{z}}^{\dagger}\right)$ breaks down to the group $\mathrm{U}(n)$, see e.g. [1, 2]. Both groups $\mathrm{O}(m)$ and $\mathrm{U}(n)$ play a fundamental role in the construction of an orthogonal basis of (Hermitean) monogenic polynomials, as explained in $[22,8,6]$.

\section{THE FISCHER DECOMPOSITION}

In 1917 Ernst Fischer proved (see [18]) that, given a homogeneous polynomial $q(X), X \in \mathbb{R}^{m}$, every homogeneous polynomial $P_{k}(X)$ of degree $k$ can be uniquely decomposed as $P_{k}(X)=Q_{k}(X)+q(X) R(X)$, where $Q_{k}(X)$ is a homogeneous polynomial of degree $k$ satisfying $q(D) Q_{k}=0, D$ being the differential operator corresponding to $X$ through Fourier identification $\left(X_{j} \leftrightarrow \partial_{x_{j}}, j=1, \ldots, m\right)$ and $R(X)$ is a homogeneous polynomial of suitable degree. If in particular $q(X)=\|X\|^{2}$, then $q(D)$ is the Laplacian $\Delta_{m}$ and $Q_{k}$ is harmonic, leading to the direct sum decomposition

$$
\mathscr{P}\left(\mathbb{R}^{m} ; \mathbb{C}\right)=\bigoplus_{k=0}^{\infty} \bigoplus_{p=0}^{\infty} r^{2 p} \mathscr{H}_{k}\left(\mathbb{R}^{m} ; \mathbb{C}\right)
$$

of the space $\mathscr{P}\left(\mathbb{R}^{m} ; \mathbb{C}\right)$ of complex valued polynomials into the spaces $\mathscr{H}_{k}\left(\mathbb{R}^{m} ; \mathbb{C}\right)$ of complex valued harmonic homogeneous polynomials of degree $k$. Each of the terms in the decomposition (1) is an irreducible $\mathrm{O}(\mathrm{m})$-module.

Euclidean Clifford analysis offers the possibility for a first refinement of this decomposition (1). Indeed, the polynomial $q(\underline{X})$ may be chosen to be $q(\underline{X})=\underline{X}$, where $\underline{X}=\sum_{\alpha=1}^{m} e_{\alpha} X_{\alpha}$ is a real vector in the complex Clifford algebra $\mathbb{C}_{m}$ constructed over $\mathbb{R}^{m}$; the differential operator $q(D)$ then is precisely the Dirac operator $\underline{\partial}=\sum_{\alpha=1}^{m} e_{\alpha} \partial_{X_{\alpha}}$ and $Q_{k}$ is a $k$-homogeneous polynomial null solution of $\underline{\partial}$, a so-called spherical monogenic. This leads to the wellknown Fischer decomposition in Euclidean Clifford analysis of the space $\mathscr{P}\left(\mathbb{R}^{m} ; \mathbb{S}\right)=\mathscr{P}\left(\mathbb{R}^{m} ; \mathbb{C}\right) \otimes \mathbb{S}$ of homogeneous polynomials taking their values in spinor space $\mathbb{S}$. This Fischer decomposition reads:

$$
\mathscr{P}\left(\mathbb{R}^{m} ; \mathbb{S}\right)=\bigoplus_{k=0}^{\infty} \bigoplus_{p=0}^{\infty} \underline{X}^{p} \mathscr{M}_{k}\left(\mathbb{R}^{m} ; \mathbb{S}\right)
$$

where $\mathscr{M}_{k}\left(\mathbb{R}^{m} ; \mathbb{S}\right)$ denotes the space of spinor valued monogenic homogeneous polynomials of degree $k$. Each of the terms in the decomposition (2) is an irreducible Pin $(m)$-module.

In particular each harmonic $k$-homogeneous polynomial $H_{k}$, be it real, complex or spinor valued, may be split in terms of homogeneous monogenic polynomials as

$$
H_{k}=M_{k}+\underline{X} M_{k-1}
$$

$M_{k}$ and $M_{k-1}$ being monogenic homogeneous polynomials of the indicated degree. This splitting also reflects the fundamental property of the Dirac operator being a "square root" of the Laplace operator: $\partial_{\underline{X}}^{2}=-\Delta$.

In its turn Hermitean Clifford analysis offers the possibility to split any monogenic homogeneous polynomial in terms of homogeneous Hermitean monogenic ones (see $[14,10])$. As explained above this is only possible in even dimension: $m=2 n$. We first decompose the space $\mathscr{P}_{k}\left(\mathbb{R}^{2 n} ; \mathbb{S}\right)$ of spinor valued $k$-homogeneous polynomials in the variables $\left(z_{1}, \ldots, z_{n}, \bar{z}_{1}, \ldots, \bar{z}_{n}\right)$ according to bidegree of homogeneity and to the homogeneous parts of spinor space:

$$
\mathscr{P}_{k}\left(\mathbb{R}^{2 n} ; \mathbb{S}\right)=\bigoplus_{a+b=k} \bigoplus_{r=0}^{n} \mathscr{P}_{a, b}^{(r)}
$$

It is interesting to know that for the cases $r=0$, respectively $r=n$, the notion of Hermitean monogenicity coincides with the notion of antiholomorphy, respectively holomorphy, in $n$ complex variables. This also explains why from now on we will restrict the spinor homogeneity degree $r$ to $1 \leq r \leq n-1$. 
If $\mathscr{M}_{a, b}\left(\mathbb{R}^{2 n} ; \mathbb{S}^{(r)}\right)$ denotes the space of Hermitean monogenic polynomials belonging to $\mathscr{P}_{a, b}^{(r)}$, then $\mathscr{M}_{k}\left(\mathbb{R}^{2 n} ; \mathbb{S}\right)$ may be decomposed into $\mathrm{U}(n)$-irreducibles as follows:

$$
\mathscr{M}_{k}=\left(\bigoplus_{a=0}^{k} \bigoplus_{r=0}^{n} \mathscr{M}_{a, k-a}^{(r)}\right) \oplus\left(\bigoplus_{a=0}^{k-1} \bigoplus_{r=1}^{n-1}\left(\frac{\underline{z}}{a+r}+\frac{\underline{z}^{\dagger}}{k-a-1+n-r}\right) \mathscr{M}_{a, k-a-1}^{(r)}\right)
$$

We went even a step further and established a Fischer decomposition theorem for the null solutions of either one of the Hermitean Dirac operators (see [7]), i.e. for the homogeneous polynomials belonging to

$$
\operatorname{Ker}_{a, b}^{r}\left(\partial_{\underline{z}}\right)=\left\{p \in \mathscr{P}_{a, b}^{r} \mid \partial_{\underline{z}} p=0\right\} \text { and } \operatorname{Ker}_{a, b}^{r}\left(\partial_{\underline{z}}^{\dagger}\right)=\left\{p \in \mathscr{P}_{a, b}^{r} \mid \partial_{\underline{z}}^{\dagger} p=0\right\}
$$

These kernel spaces are strongly related to the spaces of "initial data" needed for the Hermitean monogenic CauchyKovalevskaya extension (see the next section). We obtained the following results:

(i) Under the action of $\mathrm{U}(n)$, the space $\operatorname{Ker}_{a, b}^{r}\left(\partial_{\underline{z}}\right)$ has the multiplicity free irreducible decomposition

$$
\operatorname{Ker}_{a, b}^{r}\left(\partial_{\underline{z}}\right)=\mathscr{M}_{a, b}^{(r)} \oplus \bigoplus_{j=0}^{\min (a, b-1)}|\underline{z}|^{2 j} \underline{z}^{\dagger} \mathscr{M}_{a-j, b-j-1}^{(r-1)} \oplus \bigoplus_{j=0}^{\min (a-1, b-1)}|\underline{z}|^{2 j}\left(\underline{z^{\dagger}} \underline{z}+\frac{(a-j-1+r)}{(a+r)} \underline{z} \underline{z}^{\dagger}\right) \mathscr{M}_{a-j-1, b-j-1}^{(r)}
$$

(ii) Under the action of $\mathrm{U}(n)$, the space $\operatorname{Ker}_{a, b}^{r}\left(\partial_{\underline{z}}^{\dagger}\right)$ has the multiplicity free irreducible decomposition

$$
\operatorname{Ker}_{a, b}^{r}\left(\partial_{\underline{z}}^{\dagger}\right)=\mathscr{M}_{a, b}^{(r)} \oplus \bigoplus_{j=0}^{\min (a-1, b)}|\underline{z}|^{2 j} \underline{z} \mathscr{M}_{a-j-1, b-j}^{(r+1)} \oplus \bigoplus_{j=0}^{\min (a-1, b-1)}|\underline{z}|^{2 j}\left(\underline{z z}{ }^{\dagger}+\frac{(b-j-1+n-r)}{(b+n-r)} \underline{z}^{\dagger}\right) \mathscr{M}_{a-j-1, b-j-1}^{(r)}
$$

\section{THE CAUCHY-KOVALSKEVSKAYA EXTENSION}

The Cauchy-Kovalevskaya (CK) extension theorem (see e.g. [11, 21]) is very well known; for a nice and welldocumented historical account on this result we refer to [12]. The classical idea behind the concept of CK-extension is to characterize solutions of suitable (systems of) PDE's by their restriction, sometimes together with the restrictions of some of their derivatives, to a submanifold of codimension one. In its most simple setting the CK-extension theorem reads as follows.

Proposition 1. If the functions $F, f_{0}, \ldots, f_{k-1}$ are analytic in a neighbourhood of the origin, then the initial value problem

$$
\begin{aligned}
\partial_{t}^{k} h(\underline{x}, t) & =F\left(\underline{x}, t, \partial_{t}^{i} \partial_{\underline{x}}^{\alpha} h\right) \\
\partial_{t}^{j} h(\underline{x}, 0) & =f_{j}(\underline{x}), \quad j=0, \ldots, k-1
\end{aligned}
$$

has a unique solution which is analytic in a neighbourhood of the origin, provided that $|\alpha|+i \leq k$.

If the differential operator is chosen to be the Cauchy-Riemann operator, or more explicitly: $\partial_{t} h=-i \partial_{x} h$, with $k=1,|\alpha|=1, i=0$, it follows from Proposition 1 that a holomorphic function in an appropriate region of the complex plane is completely determined by its restriction to the real axis. For a harmonic function though, or more explicitly when $\partial_{t}^{2} h=-\partial_{x}^{2} h$, with $k=2,|\alpha|=2, i=0$, also the values of its normal derivative on the real axis should be given. In fact, there is a nice and simple construction formula for the holomorphic and harmonic CK-extensions, illustrating the necessity of these restricted values.

Proposition 2. If the function $f_{0}(x)$ is real-analytic in $|x|<a$, then

$$
F(z)=\exp \left(i y \frac{d}{d x}\right)\left[f_{0}(x)\right]=\sum_{k=0}^{\infty} \frac{1}{k !} i^{k} y^{k} f_{0}^{(k)}(x)
$$

is holomorphic in $|z|<a$ and $\left.F(z)\right|_{\mathbb{R}}=f_{0}(x)$. If moreover $f_{1}(x)$ is real-analytic in $|x|<a$, then

$$
G(z)=\sum_{j=0}^{\infty} \frac{(-1)^{j}}{(2 j) !} y^{2 j}\left(\frac{d}{d x}\right)^{2 j}\left[f_{0}(x)\right]+\sum_{j=0}^{\infty} \frac{(-1)^{j}}{(2 j+1) !} y^{2 j+1}\left(\frac{d}{d x}\right)^{2 j}\left[f_{1}(x)\right]
$$

is harmonic in $|z|<$ a and $\left.G(z)\right|_{\mathbb{R}}=f_{0}(x),\left.\frac{\partial}{\partial y} G(z)\right|_{\mathbb{R}}=f_{1}(x)$. 
The CK-extension theorem in Euclidean Clifford analysis is a direct generalization to higher dimension of this complex plane case; it reads as follows (see [3, p.110] for the version related to the Cauchy-Riemann operator instead of the Dirac operator used here).

Theorem 1. (The monogenic CK extension)

If $\widetilde{f}\left(X_{1}, X_{2}, \ldots, X_{m-1}\right)$ is real-analytic in an open set $\widetilde{\Omega}$ of $\mathbb{R}^{m-1}$ identified with $\left\{\underline{X} \in \mathbb{R}^{m}: X_{m}=0\right\}$, then there exists an open neigbourhood $\Omega$ of $\widetilde{\Omega}$ in $\mathbb{R}^{m}$ and a unique monogenic function $f$ in $\Omega$ such that its restriction to $\widetilde{\Omega}$ is precisely $\widetilde{f}$. If moreover $\widetilde{\Omega}$ contains the origin, then in an open neighbourhood of the origin this $C K$-extension $f$ is given by

$$
f\left(X_{1}, X_{2}, \ldots, X_{m}\right)=\exp \left(X_{m} e_{m} \underline{\widetilde{\partial}}\right)[\widetilde{f}]=\sum_{k=0}^{\infty} \frac{1}{k !} X_{m}^{k}\left(e_{m} \underline{\widetilde{\partial}}\right)^{k}[\widetilde{f}]
$$

where $\underline{\tilde{\partial}}$ stands for the restriction of $\underline{\partial}$ to $\mathbb{R}^{m-1}$.

Recently a CK-extension theorem for homogeneous polynomials in Hermitean Clifford analysis was obtained (see [5]). In particular, the necessary restricted values needed for a unique Hermitean monogenic extension to exist, were determined. Again we consider homogeneous polynomials with values in $\mathbb{S}^{(r)}$. We single out the variables $\left(z_{n}, \bar{z}_{n}\right)$ and consider restrictions to the vector subspace $\mathbb{C}^{n-1}=\left\{z \in \mathbb{C}^{n} \mid z_{n}=\bar{z}_{n}=0\right\}$ of codimension 1 . We may then split the value space $\mathbb{S}^{(r)}$ as

$$
\mathbb{S}^{(r)} \equiv\left(\bigwedge_{n}^{\dagger}\right)^{(r)} I=\left(\bigwedge_{n-1}^{\dagger}\right)^{(r)} I \oplus\left(\bigwedge_{n-1}^{\dagger}\right)^{(r-1)} \mathfrak{f}_{n}^{\dagger} I
$$

Hence any polynomial $p$ with values in $\left(\bigwedge_{n}^{\dagger}\right)^{(r)} I$ can be split as $p=p^{0} I+p^{1} f_{n}^{\dagger} I$, where $p^{0}$ has values in $\left(\bigwedge_{n-1}^{\dagger}\right)^{(r)}$ and $p^{1}$ has values in $\left(\bigwedge_{n-1}^{\dagger}\right)^{(r-1)}$. In [5] we proved the following CK-extension theorem.

Theorem 2. (The Hermitean monogenic CK extension)

Given as "initial data" the homogeneous polynomials $p_{a, b-j}^{0}(j=0, \ldots, b)$ with values in $\left(\bigwedge_{n-1}^{\dagger}\right)^{(r)}\left(\mathfrak{f}_{1}^{\dagger}, \ldots, f_{n-1}^{\dagger}\right)$ and $p_{a-i, b}^{1}(i=0, \ldots, a)$ with values in $\left(\bigwedge_{n-1}^{\dagger}\right)^{(r-1)}\left(\mathfrak{f}_{1}^{\dagger}, \ldots, f_{n-1}^{\dagger}\right)$, satisfying the respective compatibility conditions

$$
\begin{aligned}
& \widetilde{\partial}_{\underline{z}} p_{a, b}^{0}=0, \widetilde{\partial_{\underline{z}}} p_{a, b-1}^{0}=0, \ldots, \widetilde{\partial_{\underline{z}}} p_{a, 0}^{0}=0 \quad(r<n-1) \\
& \partial_{\underline{z}}^{\dagger} p_{a, b}^{1} f_{n}^{\dagger} I=0, \widetilde{\partial_{\underline{z}}^{\dagger}} p_{a-1, b}^{1} f_{n}^{\dagger} I=0, \ldots, \widetilde{\partial_{\underline{z}}^{\dagger}} p_{0, b}^{1} f_{n}^{\dagger} I=0 \quad(r>1)
\end{aligned}
$$

there exists a unique Hermitean monogenic homogeneous polynomial $M_{a, b}$ given by

$$
M_{a, b}=\sum_{j=0}^{b} M_{a, b-j}^{0}+\sum_{i=0}^{a} M_{a-i, b}^{1}
$$

where

$$
\begin{aligned}
& M_{a, b-j}^{0}=\bar{z}_{1}^{j} \sum_{k=0}^{A} \frac{1}{\left\lfloor\frac{k}{2}\right\rfloor !} \frac{1}{\left\lfloor\frac{k+1}{2}+j\right\rfloor !}\left(z_{n} \widetilde{\partial}_{\underline{z}} \mathfrak{f}_{n}+\bar{z}_{n} \widetilde{\partial}_{\underline{\underline{z}}}^{\dagger} f_{n}^{\dagger}\right)^{k} p_{a, b-j}^{0} I \\
& M_{a-i, b}^{1}=z_{1}^{i} \sum_{k=0}^{B} \frac{1}{\left\lfloor\frac{k}{2}\right\rfloor !} \frac{1}{\left\lfloor\frac{k+1}{2}+i\right\rfloor !}\left(z_{n} \widetilde{\partial}_{\underline{z}} \mathfrak{f}_{n}+\bar{z}_{n}{\widetilde{\partial_{\underline{z}}^{\dagger}}}_{\mathfrak{f}_{n}^{\dagger}}^{\dagger}\right)^{k} p_{a-i, b}^{1} \mathfrak{f}_{n}^{\dagger} I
\end{aligned}
$$

with $A=2(b-j)$ if $a \geq b-j$ or $A=2 a+1$ if $a<b-j$, and $B=2(a-i)$ if $b \geq a-i$ or $B=2 b+1$ if $b<a-i$, such that

$$
\begin{aligned}
\left.M_{a, b}\right|_{\mathbb{C}^{n-1}} & =p_{a, b}=p_{a, b}^{0} I+p_{a, b}^{1} \mathfrak{f}_{n}^{\dagger} I \\
\left.\frac{\partial^{i} M_{a, b}}{\partial z_{n}{ }^{i}}\right|_{\mathbb{C}^{n-1}} & =p_{a-i, b}=(-1)^{r+1} \widetilde{\partial}_{\underline{z}} p_{a-i+1, b}^{1} I+p_{a-i, b}^{1} \mathfrak{f}_{n}^{\dagger} I, \quad i=0, \ldots, a \\
\left.\frac{\partial^{j} M_{a, b}}{\partial \bar{z}_{n}{ }^{j}}\right|_{\mathbb{C}^{n-1}} & =p_{a, b-j}=p_{a, b-j}^{0} I-\mathfrak{f}_{n}^{\dagger} \widetilde{\partial_{\underline{z}}^{\dagger}} p_{a, b-j+1}^{0} I, \quad j=0, \ldots, b
\end{aligned}
$$




\section{REFERENCES}

1. F. Brackx, J. Bureš, H. De Schepper, D. Eelbode, F. Sommen, V. Souček, Fundaments of Hermitean Clifford analysis - Part I: Complex structure, Compl. Anal. Oper. Theory 1(3) (2007), 341-365.

2. F. Brackx, J. Bureš, H. De Schepper, D. Eelbode, F. Sommen, V. Souček, Fundaments of Hermitean Clifford analysis - Part II: Splitting of $h$-monogenic equations, Complex Var. Elliptic Eq. 52(10-11) (2007), 1063-1079.

3. F. Brackx, R. Delanghe, F. Sommen, Clifford Analysis, Pitman Publishers (Boston-London-Melbourne, 1982).

4. F. Brackx, H. De Schepper, D. Eelbode, V. Souček, The Howe Dual Pair in Hermitean Clifford Analysis, Rev. Mat. Iberoamericana 26(2) (2010), 449-479.

5. F. Brackx, H. De Schepper, R. Lávička, V. Souček, The Cauchy-Kovalevskaya Extension Theorem in Hermitean Clifford Analysis (submitted).

6. F. Brackx, H. De Schepper, R. Lávička, V. Souček, Orthogonal basis of Hermitean monogenic polynomials: an explicit construction in complex dimension 2. Submitted to: T.E. Simos, G. Psihoyios, Ch. Tsitouras (eds.), Numerical Analysis and Applied Mathematics, AIP Conference Proceedings, Rhodes, Greece (2010).

7. F. Brackx, H. De Schepper, R. Lávička, V. Souček, Fischer decompositions of kernels of Hermitean Dirac operators. Submitted to: T.E. Simos, G. Psihoyios, Ch. Tsitouras, Numerical Analysis and Applied Mathematics, AIP Conference Proceedings, Rhodes, Greece (2010).

8. F. Brackx, H. De Schepper, R. Lávička, V. Souček, Gel'fand-Tsetlin procedure for the construction of orthogonal bases in Hermitean Clifford analysis. Submitted to: T.E. Simos, G. Psihoyios, Ch. Tsitouras, Numerical Analysis and Applied Mathematics, AIP Conference Proceedings, Rhodes, Greece (2010).

9. F. Brackx, H. De Schepper, F. Sommen, The Hermitian Clifford analysis toolbox, Adv. Appl. Cliff. Alg., 18 (3-4), 2008, 451-487.

10. F. Brackx, H. De Schepper, V. Souček, Fischer Decompositions in Euclidean and Hermitean Clifford Analysis (submitted).

11. A. Cauchy, Oeuvres completes, série 1, Tome VII, Gauthier-Villars (Paris, 1882-1974), 17Ü-58.

12. R. Cooke, The Cauchy-Kovalevskaya Theorem (preprint, available online: http://www.cems.uvm.edu/ cooke/ckthm.pdf).

13. F. Colombo, I. Sabadini, F. Sommen, D. C. Struppa, Analysis of Dirac Systems and Computational Algebra, Birkhäuser, Boston, 2004.

14. A. Damiano and D. Eelbode, Invariant Operators Between Spaces of h-Monogenic Polynomials, Adv. Appl. Cliff. Alg. 19 (2), 2009, 237-251.

15. R. Delanghe, F. Sommen, V. Souček, Clifford Algebra and Spinor-valued Functions, Mathematics and Its Applications 53, Kluwer Academic Publishers, 1992.

16. D. Eelbode, Stirling numbers and Spin-Euler polynomials, Exp. Math. 16 (1), 2007, 55-66.

17. D. Eelbode, Irreducible $\mathfrak{s l}(m)$-modules of Hermitean monogenics, Complex Var. Elliptic Equ. 53 (10), 2008 ), $975-987$.

18. E. Fischer, Über die Differentiationsprozesse der Algebra, J. für Math. 148, 1917, 1-78.

19. J. Gilbert, M. Murray, Clifford Algebra and Dirac Operators in Harmonic Analysis, Cambridge University Press (Cambridge, 1991).

20. K. Gürlebeck, W. Sprössig, Quaternionic and Clifford Calculus for Physicists and Engineers, J. Wiley \& Sons (Chichester, 1997).

21. S. Kowalevsky, Zur Theorie der partiellen Differentialgleichung, J. für die Reine und Angew. Mathem. 80, 1875, 1 Ü-32.

22. R. Lávička, V. Souček, The Gel'fand-Tsetlin bases for spherical monogenics (submitted).

23. R. Rocha-Chavez, M. Shapiro, F. Sommen, Integral theorems for functions and differential forms in $\mathbb{C}_{m}$, Research Notes in Math. 428, Chapman\&Hall / CRC (New York, 2002).

24. I. Sabadini, F. Sommen, Hermitian Clifford analysis and resolutions, Math. Meth. Appl. Sci. 25(16-18), 2002, $1395-1414$. 